\title{
Assessing quality of life in pediatric fibrous dysplasia and McCune Albright syndrome: PEDS-QL and HADS data from the Fibrous Dysplasia Foundation Patient Registry
}

\author{
Amanda Konradi(iD
}

\begin{abstract}
Purpose: The International FD/MAS Consortium recently encouraged using the Pediatric Quality of Life Inventory (PEDS-QL) and the Hospital Anxiety and Depression scales (HADS) in clinical care. This study examines scores on these measures among pediatric fibrous dysplasia and McCune Albright (FD/MAS) patients to initiate consideration of their use in clinical treatment.

Methods: This is a retrospective analysis of pediatric data from 39 minors, ages 2-17, entered in the Fibrous Dysplasia Foundation Patient Registry from July 2016 to December 2018. Sample means and score distributions are compared to general population and chronic disease benchmarks. Associations with medical and demographic variables are also explored.

Results: Mean PEDS-QL scores for children 2-7 were inconclusive in determining at risk status for impaired quality of life (QOL). Individual score distributions suggested up to half experienced extensive physical or social impairment. Means and individual score distributions for the physical and psychosocial components of the PEDS-QL for children 817 suggested many were at risk of impaired QOL. Over half of 13-17 year-olds met the clinical benchmark for anxiety. Older males scored better than females on the PEDS-QL and HADS. Pain frequency was associated with physical function for older children.

Conclusions: Older children with FD/MAS may be more compromised in terms of psychosocial QOL than previously reported. Clinicians should be attentive to the influence of gender on QOL in older children. Online patient registries associated with rare diseases have the potential to serve as efficient and cost-effective mechanisms to jumpstart examination of new measures in consideration for clinical use.
\end{abstract}

Keywords: Fibrous dysplasia, Quality of life, Depression, Anxiety, Children, McCune Albright syndrome, PEDS-QL, HADS

\section{Plain English summary}

An international group of thought leaders concerned with the care of patients with Fibrous Dysplasia/McCune Albright Syndrome (FD/MAS) recently encouraged treating physicians to use two measures to assess children's health related quality of life (QOL), the Pediatric Quality

Correspondence: akonradi@loyola.edu

Department of Sociology, Loyola University Maryland, 4501 Charles St., Baltimore, MD 21210, USA

\section{Springer Open}

(c) The Author(s). 2021 Open Access This article is licensed under a Creative Commons Attribution 4.0 International License, which permits use, sharing, adaptation, distribution and reproduction in any medium or format, as long as you give appropriate credit to the original author(s) and the source, provide a link to the Creative Commons licence, and indicate if changes were made. The images or other third party material in this article are included in the article's Creative Commons licence, unless indicated otherwise in a credit line to the material. If material is not included in the article's Creative Commons licence and your intended use is not permitted by statutory regulation or exceeds the permitted use, you will need to obtain permission directly from the copyright holder. To view a copy of this licence, visit http://creativecommons.org/licenses/by/4.0/. 
psychological and social measures (including the PEDSQL and HADS); and to answer questions about access to and satisfaction with treatment.

The scores of children with FD/MAS were compared to published scores from the general population and from children with chronic diseases. Results showed that on average, young children, $2-7$, had significantly worse physical health than the general population, but similar psychological and social health. However, the PEDS-QL scores of $30-50 \%$ of these young children suggested they were at risk in some aspect of QOL. PEDS-QL score averages for older children, 8-17, and the distribution of individual scores in relation to published benchmarks, suggested half were at risk of impaired QOL. More than half the 13-17 year-olds also scored above 7 on the HADS, the published value for clinical levels of anxiety. Older males scored better than females on the PEDS-QL and the HADS. Pain frequency was associated with physical function for older children.

In sum, older children with FD/MAS may be more compromised in terms of psychosocial QOL than previously reported. Clinicians should be attentive to the potential influence of gender on QOL in older children. Online patient registries associated with rare diseases, such as the FDFPR, have the potential to serve as efficient and cost-effective mechanisms to jumpstart examination of new measures in consideration for clinical use.

\section{Introduction}

Fibrous dysplasia (FD) is a rare congenital and genetically heterogeneous noninheritable skeletal disease in which normal bone and marrow are replaced with discrete lesions of fibrous-osseous tissue. Radiographically, the lesions resemble ground glass. FD may affect a single bone (monostotic) or multiple bones (polyostotic) and may appear in the skull, trunk and extremities. The disease is usually diagnosed in childhood. Although not routine clinical practice, a 99Tc MDP bone scan can usually identify affected skeletal regions by 5 years of age; a head CT is used to fully assess cranial involvement [1]. Molecular genetic testing can establish the presence of the mutation in lesion tissue. Monostotic FD is most common in the rib, skull and femur; polyostotic is most common in the skull, mandible, pelvic bones and femur [2]. McCune-Albright Syndrome (MAS) "is defined as the combination of FD and one or more extra skeletal feature, OR the presence of two or more extra skeletal features," including café-au-lait skin pigmentation and endocrinopathies such as, growth hormone excess, hyperthyroidism, hypercortisolism, and renal phosphate wasting [3]. MAS is estimated to occur in 100,000 to 1 , 000,000 of the population and to comprise $5 \%$ of FD patients [4]. Bone lesions can cause functional problems, that vary depending on the bones affected, and pain [1].
FD may also result in problems of social interaction: too much attention, the wrong kinds of attention, or social exclusion [5].

The health related quality of life (QOL) of FD/MAS patients is a growing concern of medical research [5-11]. Investigators at the US National Institutes of Health (NIH), led by Dr. Michael T. Collins, explored QOL in Fibrous Dysplasia / McCune Albright Syndrome (FD/MAS) patients 14 and older with the SF-36, a self-report measure, which has 8 domains: physical functioning, role limitations due to physical problems, bodily pain, general health, energy/fatigue (also called vitality), social functioning, limitations on role performance due to emotional problems, and mental health. They measured the QOL of children diagnosed with FD with a parent proxy, the Child Health Questionnaire for children ages 7 to 13.

Collins and colleagues found that FD/MAS patients 14 and older exhibited physical QOL outcomes that were significantly lower (worse) than the general population but social-psychological measures that were similar [6]. Parent proxies for children 7 to 13 yielded scores for physical function, general health and bodily pain that were significantly lower than the general population, but social-psychological scores that were similar. In terms of physical function, children with FD were like children with asthma and rheumatoid arthritis. Children with FD had similar social-psychological scores to children with asthma, but significantly lower scores than those with rheumatoid arthritis [6]. Collins and colleagues found that children and adults with greater bone disease had poorer physical function $[6,12]$. Using the Brief Pain Inventory (BPI), a self-report measure, they found that adults and children had greater pain than the general population, that children with FD reported less pain than adults, and that reported pain did not correlate with skeletal disease burden [7].

Recently published best practice guidelines for FD care from the International FD/MAS Consortium endorsed the evaluation of QOL in FD/MAS, encouraging the use of specific measures, among them the Pediatric Quality of Life Inventory (PEDS-QL) for children and the Hospital Anxiety and Depression Scale (HADS) [3].

The purpose of this descriptive study is to analyze available pediatric data from the Fibrous Dysplasia Foundation Patient Registry (FDFPR), an online self-report portal that incorporates these two measures, to initiate consideration of their use in clinical treatment of FD/ MAS.

\section{Methods}

Study population - the fibrous Dysplasia Foundation patient registry

In June 2016, the Fibrous Dysplasia Foundation (FDF, now FD/MAS Alliance, https:fdmasalliance.org), a nonprofit 
patient advocacy organization, opened a patient registry (FDFPR, www.fdmasregistry.org/) for individuals with fibrous dysplasia and McCune Albright Syndrome, to facilitate research into this rare disease. The FDFPR consists of a battery of online surveys about the extent of a respondent's lesions and, if relevant, endocrine involvement, symptoms, surgical and medical treatments received, psychological and social measures, demographics, and questions about access to and satisfaction with treatment. The patient population consists of individuals recruited through direct email, clinicians, the Foundation website and newsletters, patient and family conferences, two FD patient-administered Facebook groups, and Twitter.

The study is a secondary analysis of deidentified FDFP $\mathrm{R}$ data. The sample consists of 39 minor patients ages 2-17, about whom demographic, bone involvement and diagnosis information was known, and relevant QOL measures were completed. FDFPR participants who did not attempt to complete the PEDS-QL or HADS, if age appropriate, were excluded. In this text, "participant" refers to the individual with FD/MAS about whom data was collected, although parents/guardians completed some of the data entry. "Self-report" refers to information specifically entered by the minor. "Parent proxy" refers to data entered by the parent/guardian about the participant.

\section{Measures}

The PEDS-QL is a generic health related QOL measure consisting of 4 core scales, physical function (8 items), emotional function (5 items), social function (5 items) and school function (5 items) that is intended for use in healthy and patient populations. Respondents are asked to recall the last month and indicate how frequently from never to almost always - they have experienced specific phenomena. Item responses (0-100) are averaged to form total and core scores; higher scores indicate higher functioning $[13,14]$. If more than $50 \%$ of a scale has been answered, missing scores may be derived from the average of existing scores.

Self-report versions of the PEDS-QL are available for ages 5-7, 8-12, 13-17 and comparable parent proxy versions are available from ages 2-17. Question wording is sustained between the versions so that responses may be compared across ages. This is desirable from a clinical perspective. Research shows alignment between selfreports and proxy reports is moderate to good, allowing for either form to be used in clinical assessment [1517]. The school function scale should only be used for descriptive or exploratory analysis with children 2-7 [13]. The PEDS-QL is responsive to clinical improvement across the physical and psychosocial scores as well as the total score [18]. Furlong and colleagues recommended the PEDS-QL for use as the generic health profile for pediatric orthopedic research [19].

There is not yet a consensus on the interpretation of PEDS scores. Initially, Varni and colleagues suggested that scores one standard deviation below healthy child means present "at risk status for Impaired HRQOL" (p332) [13]. Later, they presented findings of significant mean differences in the total scores of healthy children and those with chronic illnesses (for self-reports and parent proxies) [20]. Huang and colleagues found significant mean differences among parent proxy scores for children ages 2 to 7 and 8 to 18 and recommended use of separate age related clinical benchmarks for clinical follow up [21].

In the FDFPR, children 8 or older may complete the age-appropriate self-report version of the PEDS-QL (812, 13-18). Parents/guardians complete the proxy version for children ages 2-7 and for older children who do not complete the self-report. While either parent proxies or self-reports may be adequate for clinical assessment, they may not be aggregated for research purposes. As discussed in the analytic strategy below, self-report and parent proxy data were handled separately in this study. Score averaging was used to produce answers for three skipped questions in the data set.

The Hospital Anxiety and Depression (HADS) scale is a set of fourteen items that concern how often patients have experienced specific feelings in the past week that relate to the loss of pleasure. Answer values range from 0 to 3 and are added to produce separate anxiety and depression scores, from 0 to 21 [22, 23].

The HADS is validated on general and specific disease populations; it is used extensively in research, especially in Europe [24]. The developers have designated scores from 0 to 7 as non-clinical (normal), from 8 to 10 as mild, from 11 to 15 as moderate, and 16 or more as severe [25]. Many validation studies have affirmed the clinical cutoff of 8 , but some research on specific diseases, including cancer and chronic obstructive pulmonary disease (COPD), has suggested a need for a lower benchmark [26-28].

Children aged 13 to 17 in this study completed the HADS. No data was missing.

One item from the Brief Pain Inventory concerning how often the participant experienced pain was included for all children.

Demographics included in this study were the participant's age, gender, race, and both parent's educational attainment. Parents/guardians were directed to reference lists of bones to indicate which were affected by FD, and asked to report the patient's medical diagnosis, as monostotic FD, polyostotic FD or FD with McCune Albright Syndrome. Reports of monostotic and polyostotic disease were cross referenced with the bones listed and 
found to be consistent; reports of endocrinopathies were not cross-checked. Craniofacial disease burden was calculated from the number of zones in which FD lesions were identified in the skull, following Chen and colleagues [29].

\section{Analytic strategy}

Univariate statistics were generated to describe the sample and combined scores on the QOL measures. Bivariate analysis was conducted to establish whether demographics were significantly associated with type of diagnosis, craniofacial involvement, and type of report (self or proxy). The sample was divided by age group, 2 to 7 and 8 to 17 , and by report type to generate means for the total and core scores of the PEDS-QL. T-tests were then used to compare sample means to Varni and colleagues' normative data for self-reports and parent proxies for the general population [13], specific chronic diseases [20], and to the age specific bench marks for parent proxy scores provided by Huang and colleagues [21]. The distribution of individual scores across benchmarks was also assessed. Mean HADS results were computed and individual scores were distributed into Snaith's nonclinical, mild, moderate and serious categories [25]. ANOVAS were used to determine whether QOL scores were significantly associated with participant's diagnosis type, craniofacial involvement, frequency of pain, and gender.

\section{Results}

\section{Demographic and medical features of the sample (see Table 1)}

The 39 participants were slightly more than half male (51.3\%), overwhelmingly white (87.2\%), non-Hispanic (88.2\%), and living with parents who had the benefit of a college education (fathers $61.1 \%$ and mothers $65.8 \%$ ). Their mean age was 9.7 years and ranged from 2 to 17 . The majority had polyostotic disease (48.7\%), followed by FD/MAS (30.8\%), and monostotic disease (20.5\%). Forty six percent of the sample reported craniofacial involvement. Thirty-eight percent of them reported craniofacial involvement in one zone, $16.7 \%$ in two zones, $16.7 \%$ in three zones and $27.8 \%$ in all five zones. Chi Square tests did not reveal significant relationships between patient demographics, and diagnosis, craniofacialinvolvement, or report type.

\section{PEDS-QL}

Means were calculated for the full set of parent proxies, which included some older children, and separately for children ages $2-7$. A t-test of means revealed no significant differences for any of the core scores, so the remainder of the parent proxy analysis was conducted with 14 children ages $2-7$, who could be compared to the age graded cutoffs calculated by Huang and colleagues [21] (See Fig. 1). All mean core scores for participants ages 2-7 were below (worse than) the 2003 Health Child (203HC) norms [13], but only physical function was significantly so $(2.653, p=.02 ; 2007,-2.81$, $p=.015)$. Mean total scores for participants ages 2- to 7 were less than one standard deviation below Varni and colleagues' 2003HC norm (not meeting their criteria for "risk" of impaired health related QOL) [13]. Participant's mean total scores and physical function scores were significantly lower than Huang and colleagues' mean scores for Healthy Children ages 2-7 [21].

Close to half of the 2-7 year-olds had total PEDS-QL scores and physical function scores more than one standard deviation below Varni and colleagues 2003HC means ( $46 \%$ and $50 \%$, respectively), while almost a third (31\%) scored more than a standard deviation below healthy norms for the psychosocial domains [13] (See Table 2). More than half of the children also scored below benchmark values designated as "serious" by Huang and colleagues for the physical, emotional and social domains [21].

Results of self-reports for 18 children ages 8-17 are in Fig. 2 and Table 3. All mean core scores were significantly below Varni and colleagues' 2003HC norms for selfreports and, other than social function, also more than one standard deviation below them (meeting criteria for "risk" of impaired health related QOL) [13]. Half or more of the FD patients aged 8-17 scored more than one standard deviation below Varni and colleagues 2003HC core means, suggesting risk of impaired QOL [13].

Older children (8-17) with FD/MAS scored similarly to pediatric patients with Rheumatoid arthritis [20] on physical function, but significantly below them on all other aspects of quality of life (See Table 3). All their mean core scores were also significantly below pediatric patients with Asthma [20] (See Fig. 2).

\section{Hospital anxiety and depression scale}

HADS data was available for nineteen children ages 13 to 17 (See Table 3). Mean scores for the anxiety and depression scales were 7.37 (SD 3.96) and 2.68 (SD 2.45), respectively, less than 8 , the benchmark for clinical "caseness" and recommended follow up. Yet, among the sample, almost $58 \%$ of the participants had anxiety scores of 8 or above, meeting criteria for follow up (15.8\% in the moderate category and $42.1 \%$ in the mild category). Only one adolescent (5.3\%) had a depression score above 7 .

\section{Bivariate analysis}

Analysis of variance did not reveal a consistent directional association or significant relationship between PEDS-QL total or core scores and patient diagnosis 
Table 1 Characteristics of the Study Population

\begin{tabular}{|c|c|c|c|}
\hline Characteristics and $\mathrm{N}$ & Values & Frequency & Percentage \\
\hline \multirow[t]{2}{*}{ Gender $(N=39)$} & male & 20 & 51 \\
\hline & female & 19 & 49 \\
\hline \multirow[t]{3}{*}{ Ethnicity $(N=34)$} & Hispanic/Latino & 2 & 6 \\
\hline & Non-Hispanic & 30 & 88 \\
\hline & Ashkenazi Jewish & 2 & 6 \\
\hline \multirow[t]{2}{*}{ Race $(N=39)$} & White & 34 & 87 \\
\hline & Black and other & 5 & 13 \\
\hline \multirow[t]{3}{*}{ Mother's education ( $N=38$ ) } & High school/GED or less & 7 & 18 \\
\hline & Some college or trade school & 6 & 16 \\
\hline & College of more & 25 & 66 \\
\hline \multirow[t]{3}{*}{ Father's education $(N=36)$} & High school/GED or less & 5 & 14 \\
\hline & Some college or trade school & 9 & 25 \\
\hline & College of more & 22 & 61 \\
\hline \multirow[t]{3}{*}{ Diagnosis $(N=39)$} & monostotic FD & 8 & 21 \\
\hline & polyostotic FD & 19 & 49 \\
\hline & FD and McCune Albright & 12 & 31 \\
\hline \multirow[t]{2}{*}{ Craniofacial FD $(N=38)$} & no & 21 & 54 \\
\hline & yes & 18 & 46 \\
\hline \multirow[t]{2}{*}{ CF Zone $1(N=18)$} & no & 6 & 33 \\
\hline & yes & 12 & 67 \\
\hline \multirow[t]{2}{*}{ CF Zone $2(N=18)$} & no & 7 & 39 \\
\hline & yes & 11 & 61 \\
\hline \multirow[t]{2}{*}{ CF Zone $3(N=18)$} & no & 7 & 39 \\
\hline & yes & 11 & 61 \\
\hline \multirow[t]{2}{*}{ CF Zone $4(N=18)$} & no & 10 & 56 \\
\hline & yes & 8 & 44 \\
\hline \multirow[t]{2}{*}{ CF Zone $5(N=18)$} & no & 13 & 72 \\
\hline & yes & 5 & 28 \\
\hline \multirow[t]{5}{*}{ CF Disease Burden $(N=18)$} & 1 & 7 & 39 \\
\hline & 2 & 3 & 17 \\
\hline & 3 & 3 & 17 \\
\hline & 4 & 0 & 0 \\
\hline & 5 & 5 & 28 \\
\hline
\end{tabular}

(monostotic, polyostotic, or FD with MAS) for children 2-7 or 8-17. Similarly, craniofacial involvement was not significantly related to total scores for either age group. Neither diagnosis nor craniofacial involvement was significantly associated with adolescent's (13-17) anxiety or depression scores. Power in these analyses was low.

Reported frequency of pain correlated significantly and negatively with the PEDS-QL physical function score for children $8-17(R=-.536, p=.026)$. However, no significant relationships were found between pain and other PEDS-QL core scores for children 2-7 or 8-17. Pain also did not correlate significantly with the anxiety and depression scores of adolescents.

ANOVAs did not reveal significant gender differences in the PEDS-QL total score or core scores of participants aged 2-7 (See Table 4). Males 8-17 scored higher (better) than females $8-17$ on the total score and all core scores, of which the difference in physical function was significant $(p=.036$,$) . Mean HADS scores for females$ aged 13-17 were higher (worse) than means scores for males for both anxiety (9.13 (SD3.36) v 6.09 (SD4.01)) and depression (4.13 (SD2.36) vs 1.62 (SD2.01). The 


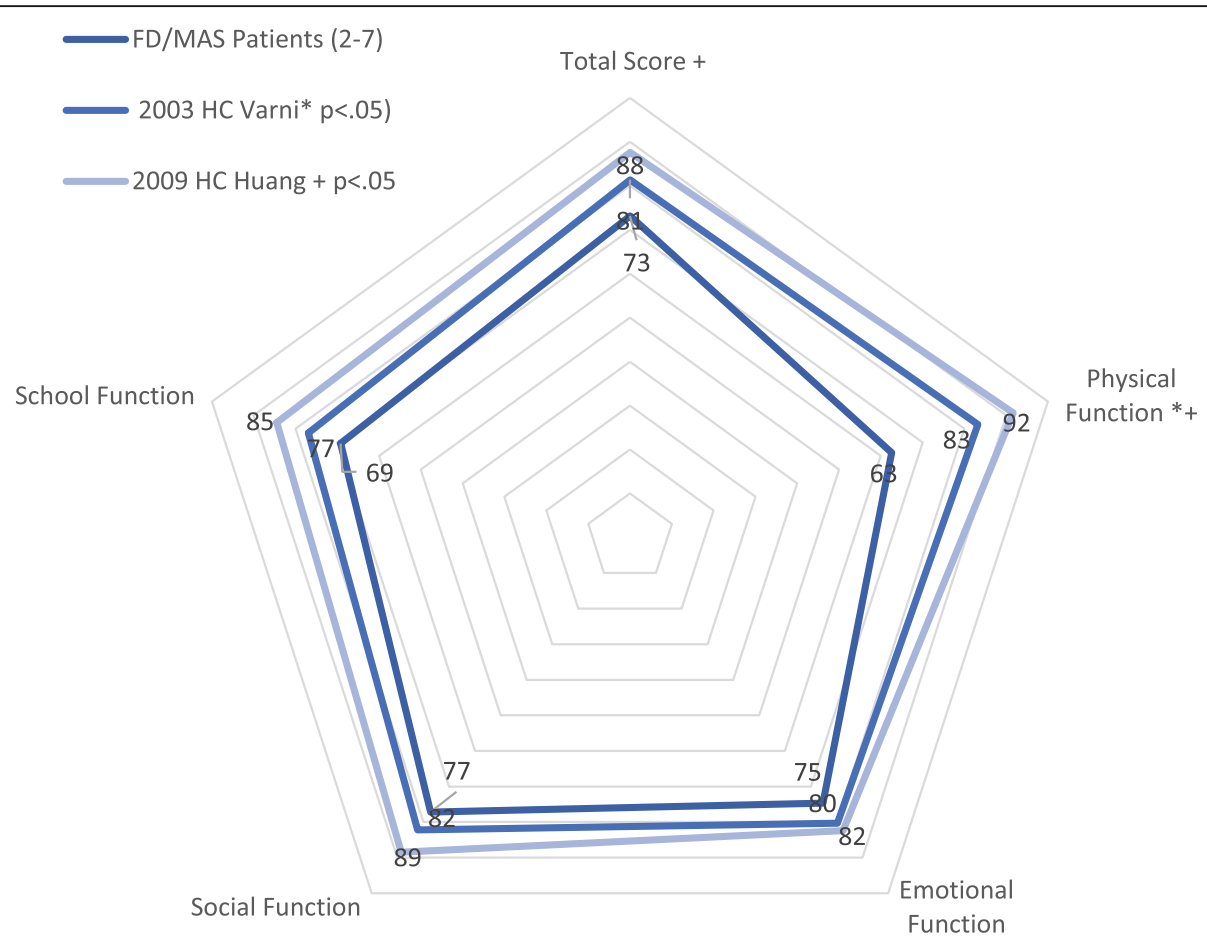

Fig. 1 PEDS-QL parent proxy ages 2-7 compared to Healthy Child norms from Varni (2003) and Huang (2009). The pentagram vertices indicate sample total and core means in relation to the two benchmarks. Significant differences $(<.05)$ are noted

difference for depression was significant $(\mathrm{F}=6.140, p=$ .024).

\section{Discussion}

The present study is the first to examine quality of life of children with FD /MAS using the PEDS-QL and HADS measures. Results from a natural history study conducted by Collins and colleagues at $\mathrm{NIH}$, involving other measures, can fruitfully be contrasted with it. Young children (2-7) with FD/MAS in this study resembled those in the natural history study, scoring below healthy norms for physical function, but similarly to healthy children on psycho-social elements [6]. Older children in this study (8-17) differed from those in the NIH study, with mean scores indicating substantially compromised QOL relative to healthy children. Yet, like the children in the natural history study, the older pediatric FD/MAS patients in this study scored similarly to children with pediatric Rheumatoid Arthritis on physical function, but comparatively worse on psychosocial components of QOL [6]. Unlike the children studied at $\mathrm{NIH}$, the scores of older FD/MAS patients indicated worse QOL than pediatric patients with Asthma [6]. Worse scores on psychosocial components of the PEDSQL in adolescents with FD/MAS than children with other chronic diseases may reflect the difficulty of managing physical symptoms of a rare condition that sets them outside age group norms without a supportive identity community [30]. Craniofacial differences, in particular, may result in difficult interactions, negative emotions, and a need to manage a stigmatic identity [31].

Collins and colleagues developed the natural history study sample from online recruitment and individuals referred to the National Institutes of Health. Participation was by invitation and reflected investigators' interests in MAS and endocrinopathies as well as bone lesions. This sample was comprised of all who volunteered. Thus, some differences described above and below may reflect differences in the samples.

These results suggest that anxiety at a level of clinical concern is common among adolescent patients (13-17) with FD/MAS, while depression is not. Compared to adolescents (ages 10-19) with bladder cancer, FD/MAS patients were more anxious (56\% versus $27 \%$ ), but had a similar rate of depression (5.9\% versus 5\%) [32]. Compared to a sample of adults with FD/MAS (unpublished results), these adolescents had lower mean scores for anxiety $(7.37$ v 8.49$)$ and depression $(2.68$ v 5.66). However, the percentage of individuals in the adult and pediatric samples who scored above 7 , indicating at least mild symptoms of anxiety were similar ( $56 \%$ v $55.6 \%$, respectively). Far fewer adolescents than adults scored above 7 for depression ( $5 \%$ vs $33.6 \%$, respectively). Considered in light of the established association between adolescent social anxiety and later depression and also greater severity of depression, a higher level of depression among adult 


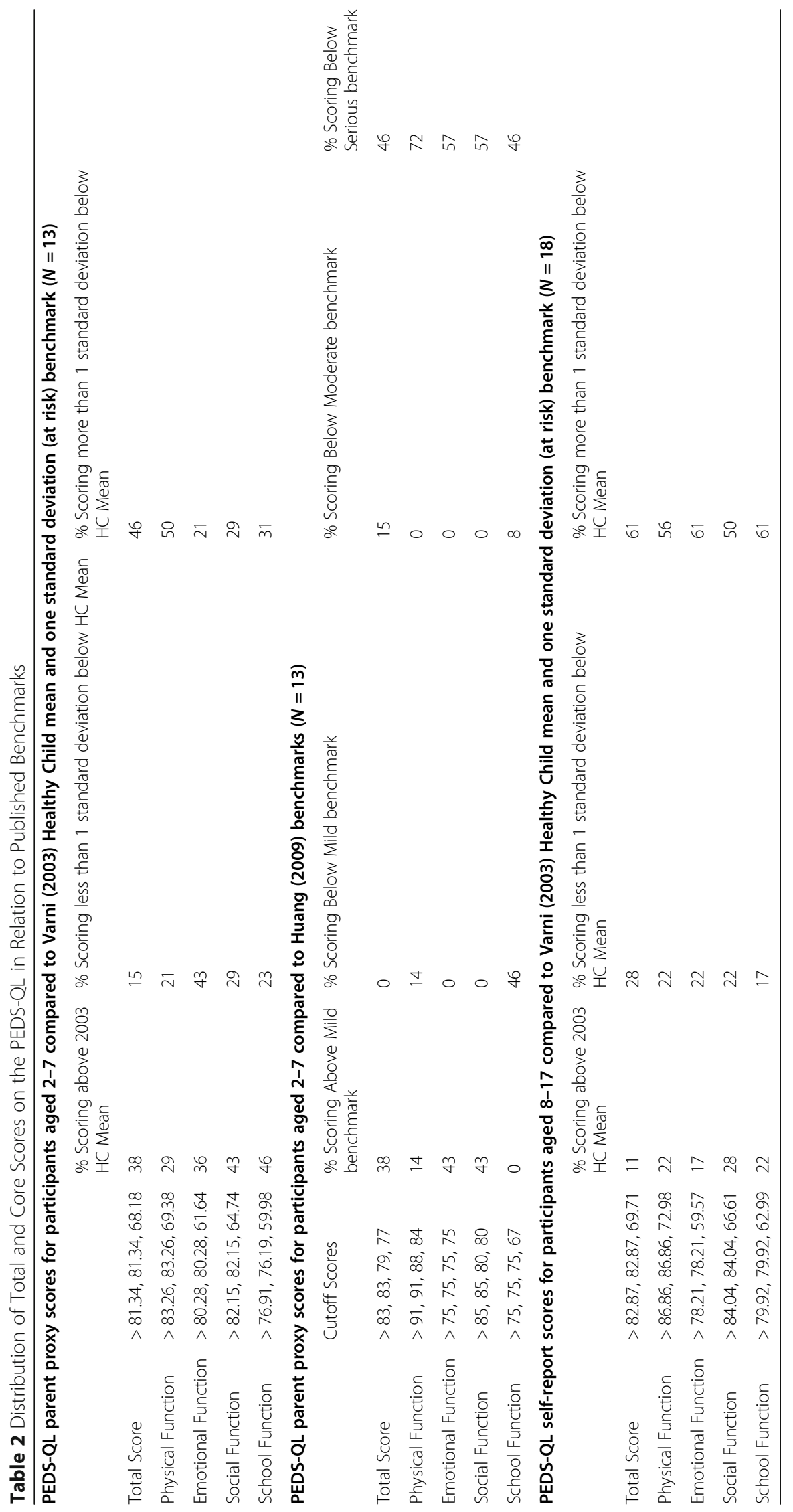




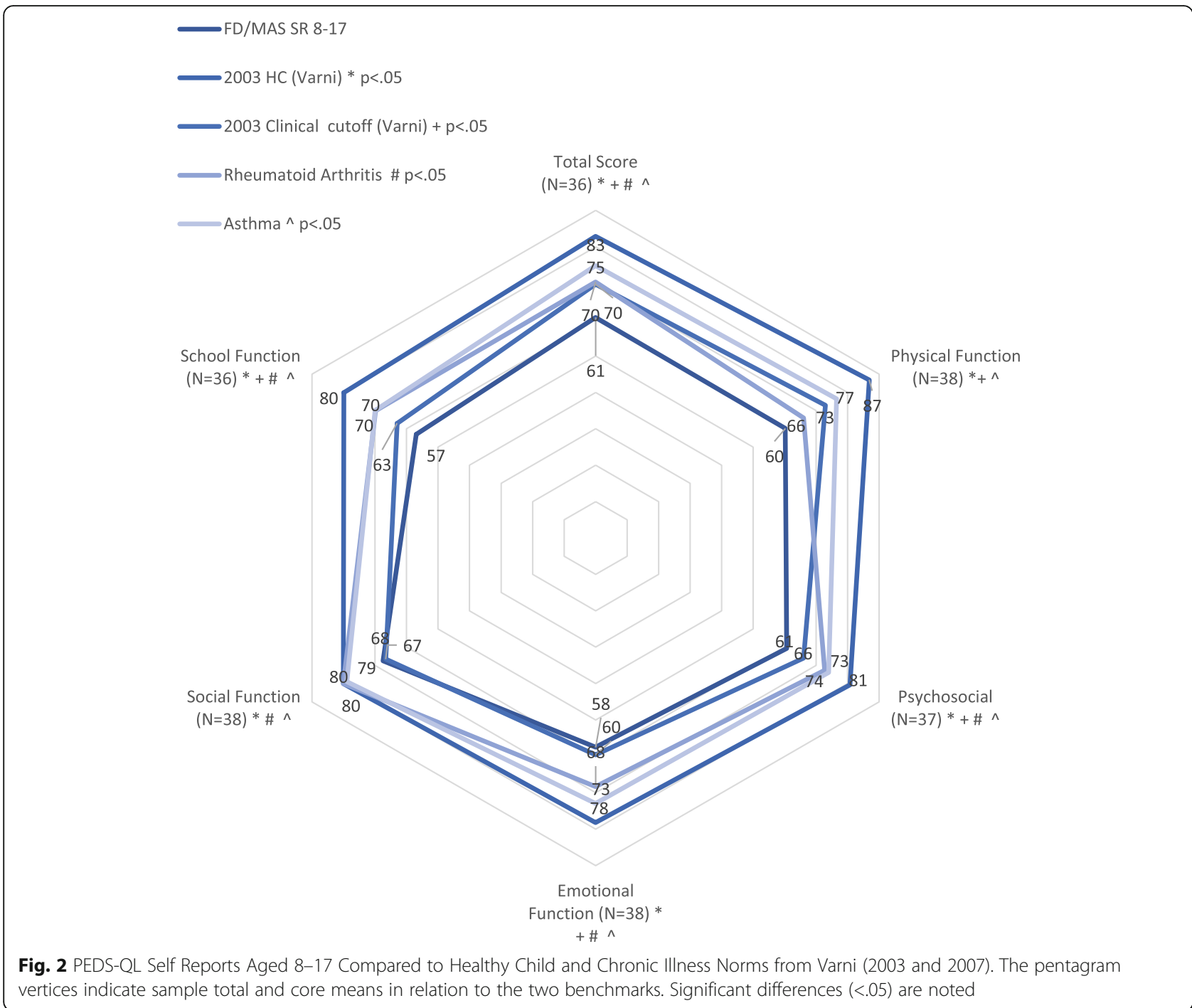

vertices indicate sample total and core means in relation to the two benchmarks. Significant differences $(<.05)$ are noted

Table 3 Hospital Anxiety and Depression Scale Individual Score Distribution Ages 13-17

\begin{tabular}{llll}
\hline Variable & Values & Frequency & $\%$ \\
\hline Anxiety & Non-clinical (0-7) & 8 & 42 \\
& Mild (8-10) & 8 & 42 \\
& Moderate (11-14) & 3 & 16 \\
& Serious (15-21) & 0 & 0 \\
Depression & & $N=19$ & \\
& Non-clinical (0-7) & 18 & 95 \\
& Mild (8-10) & 1 & 0 \\
& Moderate (11-14) & 0 & 0 \\
& Serious (15-21) & 0 & \\
\hline
\end{tabular}

FD/MAS patients supports further clinical assessment of adolescents with elevated anxiety scores [33]. Anxiety may not cause depression, but its presence in adolescence may indicate a greater propensity to develop the condition [34].

Collins and colleagues found that among children reported pain was not associated with psychosocial measures but was associated with physical function [7]. This study affirmed the lack of association with psychosocial measures and frequency of reported pain in all children, but found that the association of physical function and pain varied by age group. Variation in pain results may reflect use of pain frequency in contrast to pain intensity or differences in the nature of the reports used in this study, self-reports for older children and proxies for younger children. Alternatively, progression of disease may result in more frequent pain, or older children may experience different kinds of pain, including in the craniofacial region which is difficult to treat. 
Table 4 Gender x PEDS-QL and HADS scores ANOVA

\begin{tabular}{|c|c|c|c|c|c|c|}
\hline \multirow[t]{2}{*}{ PEDS-QL Elements } & \multicolumn{6}{|c|}{ Patients Ages 2-7 (proxy) } \\
\hline & Males & Females & $\mathrm{F}$ & $p$ value & Obs. Power & $R^{2}$ \\
\hline Total Score & 73 & 73 & .002 & .969 & .050 & .000 \\
\hline Physical Function & 69 & 56 & 612 & .450 & .111 & .053 \\
\hline Emotional Function & 82 & 71 & 1.422 & .256 & .196 & .106 \\
\hline Social Function & 74 & 79 & .147 & .710 & .164 & .012 \\
\hline \multirow[t]{3}{*}{ School Function } & 68 & 70 & .013 & .910 & .051 & .001 \\
\hline & \multicolumn{6}{|c|}{ Patients Ages 8-17 (self-report) } \\
\hline & Males & Females & $\mathrm{F}$ & $p$ value & Power & $R^{2}$ \\
\hline Total Score & 79 & 48 & 4.187 & .110 & .348 & .511 \\
\hline Physical Function & 70 & 43 & 5.295 & .036 & .576 & .261 \\
\hline Emotional Function & 62 & 50 & 1.619 & .221 & .223 & .092 \\
\hline Social Function & 72 & 62 & 1.698 & .206 & .238 & .072 \\
\hline School Function & 39 & 54 & .130 & .723 & .063 & .008 \\
\hline \multirow[t]{2}{*}{ HADS Elements } & \multicolumn{6}{|c|}{ Patients Ages 13-17 } \\
\hline & Males & Females & $\mathrm{F}$ & $p$ value & Power & $R^{2}$ \\
\hline Anxiety & 6.09 & 9.13 & 3.023 & .100 & .375 & .150 \\
\hline Depression & 1.63 & 4.13 & 6.140 & .024 & .647 & .265 \\
\hline
\end{tabular}

Research conducted in the Netherlands found a significant association between QOL measures and severity of diagnosis and craniofacial involvement in adults [11]. In contrast, this pediatric sample found neither was independently related to PEDS-QL or HADS scores. Lack of a relationship may reflect the sample size (power estimations were low) or distribution of FD/MAS types and, with respect to psychosocial scores, the distribution of craniofacial lesions in zones of the skull. It is also possible that the PEDS-QL does not capture unique features of the experience of living with FD/MAS and is inadequate to ascertain clinically important differences [35]. For example, it lacks physical function questions that address craniofacial matters and the psychosocial questions do not address stigma. Lesions that are not visible, due to their location, their inward expansion, or the extent of their development may pose less of an impediment to interaction. The age distribution in the sample may also matter to the extent that craniofacial differences among, elementary school aged children are less often negatively marked by peers than those of middle and high school aged children.

Validation studies of the PEDS-QL conducted by Varni and colleagues did not reveal gender sensitivity $[13,15]$. In contrast, among healthy Norwegian adolescents, females scored lower than males on physical function, emotional function, and the total score [36]. This study also found gender differences in QOL scores among older children, with females scoring significantly below males on physical function domain of the PEDSQL and lower on other psychosocial measures.
Some validation studies report no significant gender differences in adult HADS scores [37, 38]. Others report adult females score significantly higher on anxiety and depression than males $[39,40]$, males score higher than females [27], and that the HADS overestimates depression among women [41]. Two studies have reported significant gender differences in HADS scores among adolescents and young adults $[42,43]$, with females exhibiting higher levels of distress. This study also found adolescent females scored significantly higher than adolescent males on depression. Observed power of this analysis was comparatively high.

A gender difference in QOL among the older children may arise from their growing understanding of and adherence to gender norms. Adolescent boys may feel a need to deny physical limitations and/or the existence of negative emotions, resulting in higher scores. The loss of confidence and self-esteem that psychologists have noted adolescent girls experience in comparison to boys may contribute to their comparatively lower scores [42, 44]. It is also possible that girls experience greater social attention and possibly rejection for their deviation from health related social norms than boys do, translating into a greater perception of physical and social difficulty and lower scores on QOL measures.

This study has limitations. Disease complexity may contribute to nonsignificant results [35]. Operationalizing disease severity in terms of monostotic, polyostotic and McCune Albright syndrome, and pain in terms of frequency may have masked statistical associations. Further research using the FDFPR might seek to refine measurement of these constructs and make use of the number of bones participants report affected and the presence of specific endocrinopathies. Likewise, it might use pain intensity items from the BPI. Craniofacial involvement might also be differentiated in terms of visibility and/or functions specific to the region (sight, hearing, chewing and breathing) that may be affected by FD. The small sample size further lacks adequate power to reliably determine the importance of demographic and medical factors on QOL scores.

These results should not be interpreted as representative of all pediatric FD/MAS patients. The proportion of MAS patients in the sample is well above the estimated $5 \%$ of FD cases in the population, and the proportion of polyostotic cases in the sample is greater than monostotic cases. If disease seriousness is associated with QOL, this would tend to bias the sample toward worse scores on the PEDS-QL and HADS. While accessible to an international participation, the FDFPR is primarily comprised of individuals from the United States. The sample is not racially representative of the US population, a problem of other FD/MAS studies. It is also skewed to children of educated parents. Thus, mean scores on the 
PEDS-QOL and HADS could indicate lower levels of distress then exist in the population because the sample contains comparatively few individuals who lack access to reliable health care and/or face great social and economic obstacles for coping with a chronic illness.

The skewed demographics of the sample may reflect the greater resources that white, educated parents possess to locate the FDF and the FDFPR and to complete the lengthy battery of questions. It may also reflect a greater mistrust in medical research among members of minority populations that have been systematically neglected or damaged by prior medical research [45]. For the FDFPR to reach its potential to improve patient care, it must diversify the sample population. One avenue is to more actively recruit participants through pediatricians and general practitioners who treat underserved populations.

Research drawing from the FDFPR is further limited by its structure and scope. For example, this study uses the parent proxy of the PEDS-QL (ages 2-7) and the self-report of the PEDS-QL for ages 8-17 that are available. Because the format varies they may not be aggregated and it is not possible to ascertain whether age differences result in different scoring patterns on the PEDS-QL. Incorporating the self-report for lower ages and the proxy report for all ages would assist with resolving this issue. On the other hand, the online administration of measures in the FDFPR may encourage more accurate expressions of distress, because it reduces if not eliminates socially desirable responding [46]. The HADS was developed for in person administration and studies that have compared in person and remote administration of the HADS, via mail and online, have found higher scores in the remote samples [42, 46].

The PEDS-QL is a comprehensive measure that is valuable for detecting the clinical efficacy of treatment and is relatively short. To the extent that published norms exist for healthy children and chronically ill children, it might serve as a screening device to identify areas of trouble for individuals. Toward that end, it would be desirable to examine core scores, especially with younger children. The Total score encompasses the School Function core score, which the developers recommend only be used for descriptive or exploratory analysis in children seven and under.

The HADS was developed as a screening device and is limited to a narrow scope of depressive symptomology related to loss of the pleasure response. It does not assess feeling blue or sad and intentionally excludes some somatic symptoms $[47,48]$. From the standpoint of understanding the impact of FD/MAS on quality of life, it could result in underestimating psychological distress, especially depressive symptoms, experienced by FD/ MAS patients. The HADS has value in the treatment of FD/MAS as a diagnostic tool that leads to referral for further assessment and care and reasonably can be used in clinical appointments due to its brevity. Bearing in mind its limitations as comprehensive measure, it has value in the FDFPR because it enables comparison of the pediatric and adult populations of FD/MAS patients. Other measures should be used in research to assess the full extent of anxiety and depression in the pediatric FD/ MAS population.

Participation in the FDFPR is not limited by treatment costs or the specific interests of treating clinicians. Thus, the FDFPR could become a database that encompasses patients receiving the full scope of treatments currently in use and that documents the full range of ways patients cope with their symptoms. The FDFPR could also be a venue for FD/MAS researchers to efficiently and cost effectively study new PRO measures. Toward this end, the FDFPR needs to focus on increasing participation of children and developing recruitment strategies to diversify the participant base.

\section{Conclusions}

This study extends prior investigations of quality of life in children with FD/MAS using two instruments recommended by the International FD/MAS consortium. These results suggest that both age and gender may influence reported distress in ways not previously documented. Additionally, the results suggest the quality of life of pediatric FD/MAS patients may be more compromised than children with other chronic illnesses, suggesting a need to explore how unique qualities of the FD/MAS patient experience may result in disfunction and distress.

Monitoring the quality of life of pediatric FD/MAS patients is a desirable component of medical care. Early detection of problems in social interaction, management of emotions, in the school setting, and anxiety and depression as well as physical function will enable referral to appropriate resources. Simply raising this broad scope of issues with adolescents may increase their willingness to discuss social and psychological matters that they typically hold close and seek to manage on their own.

\footnotetext{
Abbreviations

BPI: Brief Pain Inventory; COPD: Chronic obstructive pulmonary disease; CFD: Craniofacial Fibrous Dysplasia; FD: Fibrous Dysplasia; FDF: Fibrous Dysplasia Foundation (now FD/MAS Alliance); FDFPR: Fibrous Dysplasia Foundation Patient Registry (now FD/MAS Alliance Patient Registry); FD/ MAS: Fibrous Dysplasia / McCune Albright Syndrome; HADS: Hospital Anxiety and Depression scales; MAS: McCune-Albright Syndrome; NIH: National Institutes of Health; PEDS-QL: Pediatric Quality of Life Inventory; QOL: Quality of Life; 2003HC: 2003 Healthy Child
}

\section{Acknowledgements}

Tovah Burstein generously assisted with piloting the use of data in the Fibrous Dysplasia Foundation Patent Registry. Dr. Andrea Burke collaborated to secure data from the Fibrous Dysplasia Foundation. Fibrous Dysplasia caretakers and former FD Foundation board members Lisa Heral, RN, BSN 
and Catherine Fairchild, Esq. provided useful commentary on a draft of this manuscript.

\section{Author's contributions}

Data analysis and manuscript writing was done by the author. The author(s) read and approved the final manuscript.

\section{Funding}

The Oral Maxillofacial Foundation funded acquisition of the data. No funding was received to assist with the preparation of this manuscript.

\section{Availability of data and materials}

The data that support the findings of this study are available from the Fibrous Dysplasia Foundation but restrictions apply to the availability of these data, which were used under license for the current study, and so are not publicly available. Data are however available from the authors upon reasonable request and with permission of the Fibrous Dysplasia Foundation.

\section{Declarations}

\section{Ethics approval and consent to participate}

Data collection protocols for the Fibrous Dysplasia Foundation Patient Registry (FDFPR) are reviewed by New England IRB (Needham, MA; 2018-56) and are in accordance with the 1964 Helsinki declaration and its later amendments or comparable ethical standards. Deidentified data provided for this study by the Fibrous Dysplasia Foundation was deemed exempt from further review by the Loyola University IRB.

\section{Consent for publication}

Informed consent/assent was obtained from all individual participants included in the study by the Fibrous Dysplasia Foundation.

\section{Competing interests}

The author has no relevant financial or non-financial interests to disclose.

\section{Received: 21 October 2020 Accepted: 18 March 2021}

\section{Published online: 12 April 2021}

\section{References}

1. Boyce, A. M., Florenzano, P., de Castro, L. F., \& Collins, M. T. (2015). [Updated 2019 Jun 27]. In: M. P. Adam, H. H. Ardinger, R. A. Pagon, et al., (Eds.), GeneReviews ${ }^{\circledast}$ [Internet] (pp. 1993-2020). Seattle: University of Washington, Seattle.

2. Kushchayeva, Y. S., Kushchayev, S. V., Glushko, T. Y., Tella, S. H., Teytelboym, O. M., Collins, M. T., \& Boyce, A. M. (2018). Fibrous dysplasia for radiologists: Beyond ground glass bone matrix. Insights Imaging, 9(6), 1035-1056. https:// doi.org/10.1007/s13244-018-0666-6.

3. Javaid, M. K., Boyce, A., Appelman-Dijkstra, N., Ong, J., Defabianis, P., Offiah, A., et al. (2019). Best practice management guidelines for fibrous dysplasia/ McCune-Albright syndrome: a consensus statement from the FD/MAS international consortium. Orphanet Journal of Rare Diseases, 14, 139.

4. Utriainen, P., Valta, H., Björnsdottir, S., Mäkitie, O., \& Horemuzova, E. (2018). Polyostotic fibrous dysplasia with and without McCune-Albright syndrome-Clinical features in a Nordic pediatric cohort. Frontiers in Endocrinology, 9, 96. https://doi.org/10.3389/fendo.2018.00096.

5. Konradi, A. (2017). Treating the "illness" of Cherubism and craniofacial fibrous dysplasia: Addressing the stigma of craniofacial difference. The Journal of Law, Medicine \& Ethics, 45(4), 582-595. https://doi.org/10.1177/1 073110517750598

6. Kelly, M. H., Brillante, B., Kushner, H., Gehron Robey, P., \& Collins, M. T. (2005). Physical function is impaired but quality of life preserved in patients with fibrous dysplasia of bone. Bone, 37(3), 388-394. https://doi.org/10.1016/j. bone.2005.04.026.

7. Kelly, M. H., Brillante, B., \& Collins, M. T. (2008). Pain in fibrous dysplasia of bone: Age-related changes and the anatomical distribution of skeletal lesions. Osteoporosis International, 19(1), 57-63. https://doi.org/10.1007/s001 98-007-0425-x.

8. Majoor, B. C. J., Andela, C. D., Bruggemann, J., van de Sande, M. A. J., Kaptein, A. A., Hamdy, N. A. T., ... Appelman-Dijkstra, N. M. (2017). Determinants of impaired quality of life in patients with fibrous dysplasia.
Orphanet Journal of Rare Diseases, 12(1), 80. https://doi.org/10.1186/s13023017-0629-X.

9. Majoor, B. C. J., Andela, C. D., Quispel, C. R., Rotman, M., Dijkstra, P. D. S., Hamdy, N. A. T., ... Appelman-Dijkstra, N. M. (2018). Illness perceptions are associated with quality of life in patients with fibrous dysplasia. Calcified Tissue International, 102(1), 23-31. https://doi.org/10.1007/s00223-017-0329-5.

10. Rotman, M., Andela, C. D., Majoor, B. C. J., Dijkstra, P. D. S., Hamdy, N. A. T., Kaptein, A. A., \& Appelman-Dijkstra, N. M. (2018). Passive coping strategies are associated with more impairment in quality of life in patients with fibrous dysplasia. Calcified Tissue International, 103(5), 469-475. https://doi. org/10.1007/s00223-018-0441-1.

11. Hagelstein-Rotman, M., Genders, S. W., Andela, C. D., Dijkstra, S., Majoor, B. C. J., Notting, I. C., et al. (2020). Patients With Isolated Craniofacial Dysplasia Report Better Quality of Life Compared With Those With Craniofacial Dysplasia and Extracranial Involvement. Ophthalmic Plastic and Reconstructive Surgery, 36, 292-297.

12. Collins, M. T., Kushner, H., Reynolds, J. C., Chebli, C., Kelly, M. H., Gupta, A., .. Chen, C. C. (2004). An instrument to measure skeletal burden and predict functional outcome in fibrous dysplasia of bone. Journal of Bone and Mineral Research, 20(2), 219-226. https://doi.org/10.1359/JBMR.041111.

13. Varni, J. W., Burwinkle, T. M., Seid, M., \& Skarr, D. (2003). The PedsQL ${ }^{\text {TM* }} 4.0$ as a pediatric population health measure: Feasibility, reliability, and validity. Ambulatory Pediatrics, 3(6), 329-341. https://doi.org/10.1367/1539-4409(2003 003<0329:TPAAPP > 2.0.CO;2

14. Hullmann, S. E., Ryan, J. L., Ramsey, R. R., Chaney, J. M., \& Mullins, L. L. (2011). Measures of general pediatric quality of life: Child health questionnaire (CHQ), DISABKIDS chronic generic measure (DCGM), KINDL-R, pediatric quality of life inventory (PedsQL) 4.0 generic Core scales, and quality of my life questionnaire (QoML). Arthritis Care Research, 63(S11), S420-S430. https:// doi.org/10.1002/acr.20637.

15. Varni, J. W., Limbers, C. A., \& Burwinkle, T. M. (2007). Parent proxy-report of their children's health-related quality of life: an analysis of 13,878 parents' reliability and validity across age subgroups using the PedsQL ${ }^{\text {TM }} 4.0$ Generic Core Scales. Health and Quality of Life Outcomes, 5, 2.

16. Lee, Y., Yang, H.-J., Chen, V. C., Lee, W.-T., Teng, M.-J., Lin, C.-H., et al. (2016) Meta-analysis of quality of life in children and adolescents with ADHD: By both parent proxy-report and child self-report using PedsQL ${ }^{\top M}$. Research in Developmental Disabilities, 51-52, 160-172.

17. Panepinto, J. A., Pajewski, N. M., Foerster, L. M., \& Hoffmann, R. G. (2008). The Performance of the PedsQL Generic Core Scales in Children With Sickle Cell Disease. Journal of Pediatric Hematology/Oncology, 30, 666-673.

18. Desai, A. D., Zhou, C., Stanford, S., Haaland, W., Varni, J. W., \& MangioneSmith, R. M. (2014). Validity and Responsiveness of the Pediatric Quality of Life Inventory (PedsQL) 4.0 Generic Core Scales in the Pediatric Inpatient Setting. JAMA Pediatrics, 168, 1114.

19. Furlong, W., Barr, R. D., Feeny, D., \& Yandow, S. (2005). Patient-focused measures of functional health status and health-related quality of life in pediatric orthopedics: A case study in measurement selection. The Cleft Palate-craniofacial Journal, 3 [cited 1AD Jan 1]. BioMed Central, 3-15.

20. Varni, J. W., Limbers, C. A., \& Burwinkle, T. M. (2007). Impaired health-related quality of life in children and adolescents with chronic conditions: a comparative analysis of 10 disease clusters and 33 disease categories/ severities utilizing the PedsQL ${ }^{\mathrm{TM}} 4.0$ Generic Core Scales. Health and Quality of Life Outcomes, 5, 43.

21. Huang, I.-C., Thompson, L. A., Chi, Y.-Y., Knapp, C. A., Revicki, D. A., Seid, M., \& Shenkman, E. A. (2009). The linkage between pediatric quality of life and health conditions: Establishing clinically meaningful cutoff scores for the PedsQL. Value in Health, 12(5), 773-781. https://doi.org/10.1111/j.1524-4733.2008.00487.x.

22. Zigmond, A. S., \& Snaith, R. (1983). The hospital anxiety and depression scale. Acta Psychiatrica Scandinavica, 67(6), 361-370. https://doi.org/10.1111/ j.1600-0447.1983.tb09716.x

23. Snaith, R. P. (2003). The hospital anxiety and depression scale. Health and Quality of Life Outcomes, 4, 29-34

24. Cosco, T. D., Doyle, F., Ward, M., \& McGee, H. (2012). Latent structure of the hospital anxiety and depression scale: A 10-year systematic review. Journal of Psychosomatic Research, 72(3), 180-184. https://doi.org/10.1016/j. jpsychores.2011.06.008.

25. Snaith, R. P., \& Zigmond, A. S. (1994). The hospital anxiety and depression scale manual. Windsor: NFER-Nelson.

26. Singer, S., Kuhnt, S., Götze, H., Hauss, J., Hinz, A., Liebmann, A., ... Schwarz, R. (2009). Hospital anxiety and depression scale cutoff scores for cancer 
patients in acute care. British Journal of Cancer, 100(6), 908-912. https://doi org/10.1038/sj.bjc.6604952.

27. Nowak, C., Sievi, N. A., Clarenbach, C. F., Schwarz, E. I., Schlatzer, C., Brack, T., et al. (2014). Accuracy of the hospital anxiety and depression scale for identifying depression in chronic obstructive pulmonary disease patients. Pulmonary Medicine, 2014, 1-7.

28. Bjelland, I., Dahl, A. A., Haug, T. T., \& Neckelmann, D. (2002). The validity of the hospital anxiety and depression scale an updated literature review. Journal of Psychosomatic Research, 52(2), 69-77. https://doi.org/10.1016/ S0022-3999(01)00296-3.

29. Chen, Y. R., \& Noordhoof, M. S. (1990). Treatment of craniomaxillofacial fibrous dysplasia: How early and how extensive? Plastic and Reconstructive Surgery, 86(5), 835-842. https://doi.org/10.1097/00006534-199011000-00002.

30. Roberts, C. M., Gamwell, K. L., Baudino, M. N., Grunow, J. E., Jacobs, N. J., Tung, J., et al. (2020). The Contributions of Illness Stigma, Health Communication Difficulties, and Thwarted Belongingness to Depressive Symptoms in Youth with Inflammatory Bowel Disease. Journal of Pediatric Psychology, jsz084. 45, 81-90.

31. Thompson, A., Kent, G., Topolski, T. D., Edwards, T. C., \& Patrick, D. L. (2005). Adjusting to disfigurement: processes involved in dealing with being visibly different. The Cleft Palate-craniofacial Journal, 21, 42 Elsevier Ltd.

32. Kabra, A. T., Feustel, P. J., \& Kogan, B. A. (2015). Screening for depression and anxiety in childhood neurogenic bladder dysfunction. Journal of Pediatric Urology, 11, 75.e1-75.e7.

33. Kraines, M. A., White, E. J., Grant, D. M., \& Wells, T. T. (2019). Social anxiety as a precursor for depression: Influence of interpersonal rejection and attention to emotional stimuli. Psychiatry Research, 275, 296-303. https://doi. org/10.1016/j.psychres.2019.04.001.

34. Rice, F., van den Bree, M. B., \& Thapar, A. (2004). A population-based study of anxiety as a precursor for depression in childhood and adolescence. BMC Psychiatry, 4(1), 43. https://doi.org/10.1186/1471-244X-4-43.

35. Guyatt, G. H., Osoba, D., Wu, A. W., Wyrwich, K. W., \& Norman, G. R. (2002). Methods to explain the clinical significance of health status measures. Mayo Clinic Proceedings, 77(4), 371-383. https://doi.org/10.4065/77.4.371.

36. Reinfjell, T., Hjemdal, O., Aune, T., Vikan, A., \& Diseth, T. H. (2008). The pediatric quality of life inventory (PedsQL ${ }^{\mathrm{TM}}$ ) 4.0 as an assessment measure for depressive symptoms: A correlational study with young adolescents. Nordic Journal of Psychiatry, 62(4), 279-286. https://doi.org/10.1080/080394 80801983950.

37. Bocéréan, C., \& Dupret, E. (2014). A validation study of the hospital anxiety and depression scale (HADS) in a large sample of French employees. BMC Psychiatry, 14(1), 354. https://doi.org/10.1186/s12888-014-0354-0.

38. Hunt-Shanks, T., Blanchard, C., Reid, R., Fortier, M., \& Cappelli, M. (2010). A psychometric evaluation of the hospital anxiety and depression scale in cardiac patients: Addressing factor structure and gender invariance. British Journal of Health Psychology, 15(1), 97-114. https://doi.org/10.1348/13591 0709X432745.

39. Crawford, J. R., Henry, J. D., Crombie, C., \& Taylor, E. P. (2001). Normative data for the HADS from a large non-clinical sample. British Journal of Clinical Psychology, 40(4), 429-434. https://doi.org/10.1348/014466501163904.

40. Mirani, S. H., Areja, D., Gilani, S. S., Tahir, A., Pathan, M., \& Bhatti, S. (2019). Frequency of depression and anxiety symptoms in surgical hospitalized patients. Cureus, 11, e4141.

41. Macêdo, E. A., Appenzeller, S., \& Costallat, L. T. L. (2018). Depression in systemic lupus erythematosus: Gender differences in the performance of the Beck depression inventory (BDI), Center for Epidemiologic Studies Depression Scale (CES-D), and hospital anxiety and depression scale (HADS). Lupus, 27(2), 179-189. https://doi.org/10.1177/0961203317713142.

42. Jörngården, A., Wettergen, L., \& von Essen, L. (2006). Measuring healthrelated quality of life in adolescents and young adults: Swedish normative data for the SF-36 and the HADS, and the influence of age, gender, and method of administration. Health and Quality of Life Outcomes, 4(1), 91. https://doi.org/10.1186/1477-7525-4-91.

43. White, D., Leach, C., Sims, R., Atkinson, M., \& Cottrell, D. (1999). Validation of the hospital anxiety and depression scale for use with adolescents. The British Journal of Psychiatry, 175(5), 452-454. https://doi.org/10.1192/bjp.175. 5.452 .

44. Quatman T, Watson C. Gender differences in adolescent self-esteem: An exploration of domains. J The Journal of Genetic Psychology 2001;162:93117, 1, DOI: https://doi.org/10.1080/00221320109597883.
45. Skloot, R. (2010). The immortal life of Henrietta lacks. New York: Crown Publishing Group.

46. McCue, P., Buchanan, T., \& Martin, C. R. (2006). Screening for psychological distress using internet administration of the hospital anxiety and depression scale (HADS) in individuals with chronic fatigue syndrome. British Journal of Clinical Psychology, 45(4), 483-498. https:/doi.org/10.1348/014466505X82379.

47. Saez-Flores, E., Tonarely, N. A., Barker, D. H., \& Quittner, A. L. (2018). Examining the stability of the hospital anxiety and depression scale factor structure in adolescents and young adults with cystic fibrosis: A confirmatory factor analysis. Journal of Pediatric Psychology, 43(6), 625-635. https://doi.org/10.1093/jpepsy/jsx155.

48. Coyne, J. C., \& van Sonderen, E. (2012). The hospital anxiety and depression scale (HADS) is dead, but like Elvis, there will still be citings. Journal of Psychosomatic Research, 73(1), 77-78. https://doi.org/10.1016/j.jpsychores.2 012.04.002.

\section{Publisher's Note}

Springer Nature remains neutral with regard to jurisdictional claims in published maps and institutional affiliations.

\section{Submit your manuscript to a SpringerOpen ${ }^{\circ}$ journal and benefit from:}

- Convenient online submission

- Rigorous peer review

- Open access: articles freely available online

- High visibility within the field

- Retaining the copyright to your article

Submit your next manuscript at $\boldsymbol{\nabla}$ springeropen.com 